\title{
Transition of medical records from paper to electronic records - implications for Out-of- Hospital Cardiac Arrest registration
}

Stig Nikolaj Blomberg', Freddy Lippert', Fredrik Folke', Helle Collatz Christensen'

${ }^{1}$ Emergency Medical Services Copenhagen, University of Copenhagen, Denmark

\section{BACKGROUND}

Copenhagen EMS changed to electronic records in ambulances in May 2015. Henceforth all registrations of Out-of-Hospital Cardiac Arrest (OHCA) are documented as an integrated part of the electronic medical record. Previously OHCA was documented by EMS-personnel filling in and submitting a paper-record to the cardiac registry. We investigated consequences in respect to incidence and completeness of registrations before and after the transition.

\section{METHODS}

A total of 8,010 records regarding OHCA were retrospectively reviewed. The paper record group consisted of 3.481 records for all OHCA collected nationally 1 January 2016 to 31 December 2016. The historic cohort has been reported. (1) In the electronic record group for 1 January 2016 to 31 December 2018 a total of 4,529 records were eligible for review. All electronic records were validated. Endpoints were incidence and completeness.
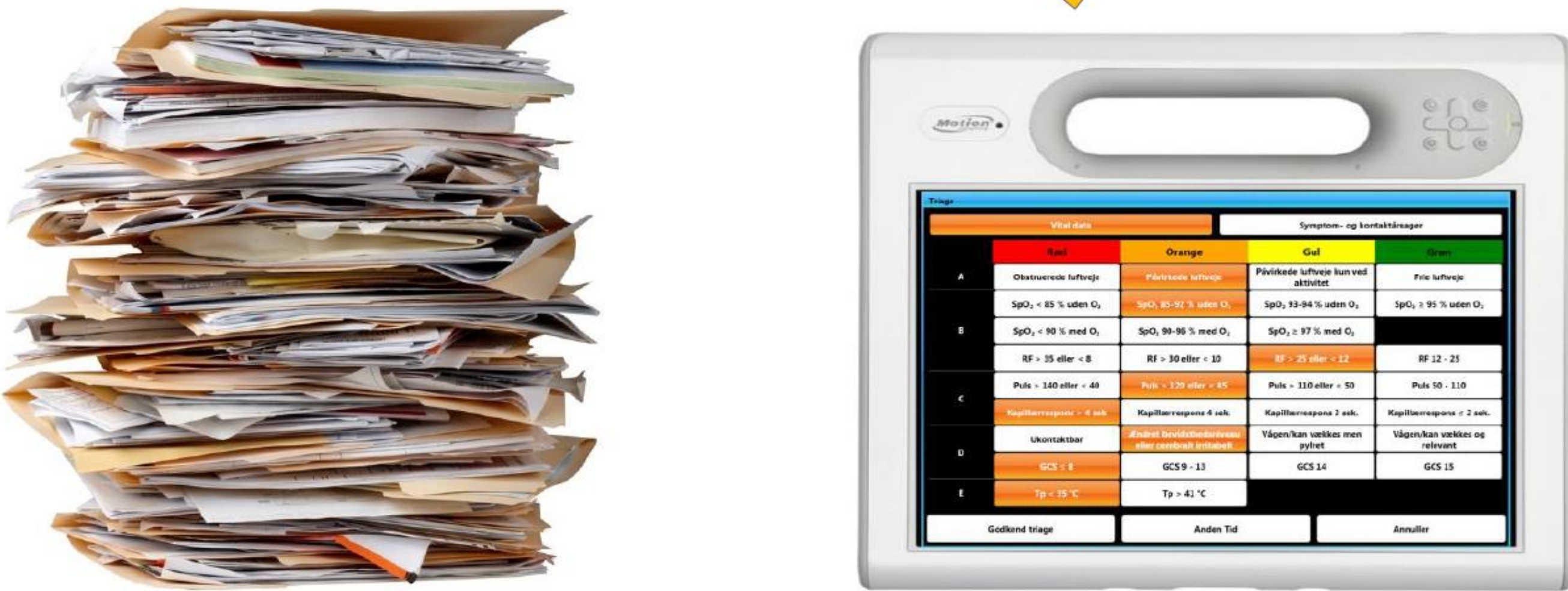

Tablet used for the new electronic record including registration of Out-of-hospital Cardiac Arrest

\section{RESULTS}

The incidence of reported OHCA was significantly lower in paper records compared to electronic records (61 vs 85 per 100,000 inhabitants). A declining incidence was observed in the years prior to 2016, explained by receding attention to the paper records as the electronic records being implemented, though the protocol for reporting OHCA on paper did not change.

Characteristics of OHCA was similar in the two samples. A significantly higher completeness of datapoints in the electronic records was observed (5.7\% vs $10.6 \%)$. Validation of data from the medical records revealed cases of OHCA not previously reported. These were patients successfully resuscitated prior to arrival of EMS-personnel needing no further treatment or cases with irreversible signs of death, where EMS-personnel attempted resuscitation.

Proportion of ROSC, OHCA in private home, prior CPR and male patients 2016 paper and 2016-2018 electronic records

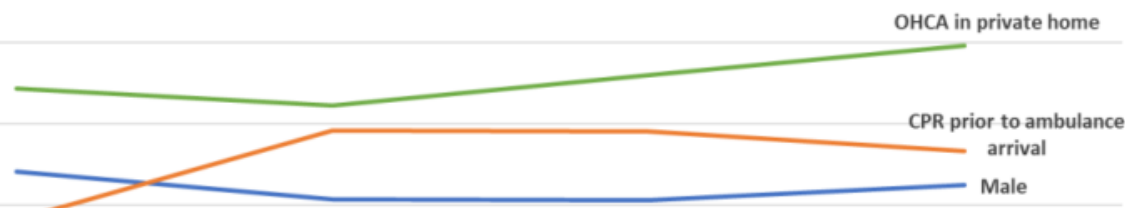

\begin{tabular}{|c|c|c|c|c|}
\hline Value & $\begin{array}{c}\text { Paperbased } 2016 \\
\text { Denmark nationwide }\end{array}$ & $\begin{array}{l}\text { Electronic records } \\
2016 \text { Copenhagen }\end{array}$ & $\begin{array}{l}\text { Electronic } \\
\text { records } 2017 \\
\text { Copenhagen }\end{array}$ & $\begin{array}{c}\text { Electronic } \\
\text { records } 2018 \\
\text { Copenhagen }\end{array}$ \\
\hline Entries & & 1500 & 1539 & 1490 \\
\hline missing person IDpnumber & & 80 & 87 & 78 \\
\hline $\mathrm{OHCA}, \mathrm{n}$ & 3481 & 1420 & 1452 & 1412 \\
\hline Median age & 72 & 73 & 73 & 74 \\
\hline IQR $25 \%-75 \%$ & $(62-82)$ & $(62-83)$ & $(62-82)$ & $(62-83)$ \\
\hline Male & 71 & 71 & 71 & 72 \\
\hline IQR $25 \%-75 \%$ & $(61-80)$ & $(59-80)$ & $(59-80)$ & $(60-80)$ \\
\hline Female & 75 & 78 & 77 & 77 \\
\hline IQR $25 \%-75 \%$ & $(65-85)$ & $(67-86)$ & $(66-85)$ & $(65-86)$ \\
\hline Male, $n$ & 2230 & 862 & 879 & 881 \\
\hline (\%) & $64,10 \%$ & $60,70 \%$ & $60,60 \%$ & $62,39 \%$ \\
\hline OHCA in private home & 2532 & 1026 & 1103 & 1123 \\
\hline (\%) & $74,30 \%$ & $72,25 \%$ & $75,96 \%$ & $79,53 \%$ \\
\hline Witnessed & 1278 & 697 & 683 & 622 \\
\hline (\%) & $37,00 \%$ & $49,08 \%$ & $47,04 \%$ & $44,05 \%$ \\
\hline EMS-witnessed & 463 & 139 & 139 & 137 \\
\hline (\%) & $13,40 \%$ & $9,79 \%$ & $9,57 \%$ & $9,70 \%$ \\
\hline Unwitnessed OHCA & 1711 & 584 & 630 & 653 \\
\hline (\%) & $49,60 \%$ & $41,13 \%$ & $43,39 \%$ & $46,25 \%$ \\
\hline Shockable rythm & 577 & 242 & 227 & 211 \\
\hline (\%) & $17,70 \%$ & $18,22 \%$ & $16,19 \%$ & $15,90 \%$ \\
\hline ROSC & 679 & 410 & 377 & 372 \\
\hline (\%) & $21,50 \%$ & $28,87 \%$ & $25,96 \%$ & $26,35 \%$ \\
\hline CPR prior to ambulance arrival & 2027 & 981 & 1003 & 941 \\
\hline (\%) & $58 \%$ & $69,08 \%$ & $69,08 \%$ & $66,64 \%$ \\
\hline
\end{tabular}

Table 1: Characteristics of OHCA-registrations with respectively paperbased registrations and electronic medical records.

\section{CONCLUSION:}

Using electronic records significantly increased recorded incidence of OHCA. A large group of previously not registrered $\mathrm{OHCA}$ were identified. 